\title{
Natural Patterns of Neural Activity \\ How Physiological Mechanisms are Orchestrated to Cope with Real Life
}

\section{Rafael Kurtz* and Martin Egelhaaf}

\author{
Lehrstuhl für Neurobiologie, Fakultät für Biologie, Universität Bielefeld, \\ Postfach 1001 31, D-33501 Bielefeld, Germany
}

\begin{abstract}
Physiological mechanisms of neuronal information processing have been shaped during evolution by a continual interplay between organisms and their sensory surroundings. Thus, when asking for the functional significance of such mechanisms, the natural conditions under which they operate must be considered. This has been done successfully in several studies that employ sensory stimulation under in vivo conditions. These studies address the question of how physiological mechanisms within neurons are properly adjusted to the characteristics of natural stimuli and to the demands imposed on the system being studied. Results from diverse animal models show how neurons exploit natural stimulus statistics efficiently by utilizing specific filtering capacities. Mechanisms that allow neurons to adapt to the currently relevant range from an often immense stimulus spectrum are outlined, and examples are provided that suggest that information transfer between neurons is shaped by the system-specific computational tasks in the behavioral context.

Index Entries: Adaptation; calcium imaging; cricket cercal system; dendritic processing; in vivo stimulation; motion vision; photoreceptor; sensory coding; synaptic transmission; temporal filtering.
\end{abstract}

\section{Introduction}

The diversity of intricate mechanisms of neuronal signaling is usually most easily studied in reduced preparations rather than in the

\footnotetext{
* Author to whom all correspondence and reprint requests should be addressed. E-mail: rafael.kurtz@biologie.uni-bielefeld.de
}

intact animal. Thorough investigations of the physiological processes that regulate the firing of neurons are conventionally performed on slices of neuronal tissue that properly fit into a Petri dish or recording chamber. Responses of sensory cells and neurons can sometimes be analyzed in detail only when the cells are extracted from the layers of tissue that normally surround them. For instance, synaptic transmission has often been investigated in 
preparations of the neuromuscular junction, where the muscle was prevented from twitching (and thus from potentially causing mechanical artefacts in the recording) (1-3). Photoreceptor light adaptation is frequently studied in isolated rods or cones that are amenable to electrophysiological recording and pharmacological manipulation, but no longer interact with neighboring cells (4-6).

During the past few decades, a vast number of in vitro studies on various preparations provided detailed insight into complicated molecular signaling pathways, and several specific aspects of neuronal excitability could even be traced to the properties of particular types of ion channels (7-9). Nevertheless, it is advisable to look beyond in vitro preparations. When the nerve cells being investigated are deprived of their natural input, neuronal excitation must be evoked artificially-for example, by the injection of current or by the application of neurotransmitters or appropriate agonists. However, such an artificial stimulation results in ranges and temporal patterns of neuronal activity that do not always match those that occur naturally. In order to draw conclusions about how neurons and sensory cells operate under real-life conditions, it is essential to complement in vitro studies with investigations performed on animals that are as intact as possible and can be provided with sensory input.

Ideally, these sensory stimuli should be identical to those encountered by the behaving animal in its natural surroundings. However, such stimulation is often not compatible with experimental procedures. Therefore, the in vivo studies of neurophysiological mechanisms that are the topic of this article differ in the extent to which the stimulation approximates natural conditions, although in all examples sensory stimulation replaces artificial excitation. Depending on the type of preparation, the techniques applied and the questions addressed, the sensory stimuli range from simple, typical "laboratorydesigned" ones to stimuli resembling the complex ones experienced by the behaving animal as closely as possible.
This review can only glimpse at the wealth of approaches pursued in recent years to elucidate how neural mechanisms operate under natural conditions. Furthermore, our survey focuses on sensory circuits, leaving out investigations of how motor patterns are generated under real-life conditions. Three fundamental questions are addressed in this article. First, how is sensory processing organized within the constraints imposed by the system's natural input? Second, which adaptational mechanisms are implemented to cope with the wide spectrum of stimulus strengths an animal encounters in behavioral situations? Third, how is synaptic transfer of sensory information matched to the particular tasks of the system?

\section{Sensory Coding}

The processing of sensory information of different modalities has been investigated in a wide range of animal models. In this article, we present examples from three animal models-electric fish, crickets, and flies-covering the sensory modalities of electroreception, mechanosensation, and vision. However different, all these studies share one important aspect: sensory coding mechanisms were studied during presentation of sensory stimuli, but not during artificial excitation of the sensory receptors. The electrosensory system of certain species of fish is one of the first systems in which neurophysiological investigations were performed during natural stimulation. Since this topic was recently highlighted in a series of review papers (10), we will only briefly mention some aspects of electrosensory information processing. Weakly electric fish explore their surroundings by active electrolocation. They generate an electric field by means of electric organ discharges and sense perturbations of the electric field caused by objects or conspecifics with an array of electroreceptor organs distributed on their skin. This process enables electric fish to perceive an image of their surroundings. The fact that electrosen- 
sory input patterns largely depend on the behavior of the fish itself-namely its electric organ discharges-emphasizes the need to analyze the responses of neurons during natural stimulation. Therefore, the research on electric fish provides early examples of behavioral and neuronal responses observed during playback of sensory stimuli, which were generated previously by the behaving animal and recorded by the experimenter (11-13).

Several years ago, in vivo patch clamp recordings of electrosensory neurons became feasible, and now allow a detailed analysis of the physiological mechanisms underlying electrosensory information processing (14). In the midbrain of Eigenmannia, there are biophysically distinct groups of electrosensory neurons that respond best to different ranges of frequencies of the electric fields. This selectivity may be crucial for discerning small object-induced perturbations of the electric field against a high level of background noise. Frequency-selective neuronal responses also allow adjustment of the frequency at which the electric organ discharges. This adjustment is necessary to avoid interference from discharges of neighboring fish, and is known to be significant in the context of communication during reproductive behavior (10). Neurons respond in a frequency-selective way by acting either as low-, band-, or high-pass filters. The operating mode is shaped by passive membrane characteristics and dendritic morphology. In addition, the expression of voltage-dependent conductances may contribute to the temporal properties of electrosensory midbrain neurons (15).

A similar function for active conductances in providing neurons with specific filtering properties was found to exist in the visual system of flies (16). Large, motion-sensitive neurons, known as tangential cells (TCs), sample motion information from retinotopically arranged input elements (17-19) (Fig. 1A). Thus, they respond in a directionally selective manner to motion in a large part of the visual surroundings. Because of their intricate receptive field properties, some TCs have been sug- gested to respond specifically to the optic flow associated with certain types of self-motion (20-22), whereas others detect movements of objects against a steady background (23-25).

Approximately 60 TCs can be individually identified, and intracellular recordings can be obtained from some of them during sensory stimulation in vivo. Depending on cell type, TCs respond to visual motion by graded shifts of their membrane potential, by spikelike events superimposed on graded membranepotential shifts, or by large-amplitude action potentials. The different response modes are the result of active conductances with which some of the TCs are equipped (26). These conductances also specify how TCs filter their visual input. Two classes of TCs differ in their dynamic response properties when tested with sinusoidally modulated velocities (16). One class possesses mainly passive membrane conductances. Its membrane potential follows slow fluctuations in image velocity, whereas rapid fluctuations are filtered out. In contrast, the response of the other class of TCs at higher frequencies is boosted by means of a voltagegated sodium conductance, allowing the neuron to transmit information about somewhat faster changes in image motion $(16,27)$. However, upon inactivation of this conductance, the filtering properties of the two TC classes become very similar.

The magnitude of membrane-potential responses of the previously mentioned two classes of TCs depends in a similar way on the velocity and the direction of constant velocity motion. However, this does not always indicate that their output information is identical. This point was addressed by measuring concentration changes of cytosolic calcium in the two classes of TCs during visual stimulation (28). One of the two TC classes possesses dendritic output synapses (29). The magnitude of dendritic calcium accumulation differs significantly in its stimulus dependency between the TC classes, although that of the corresponding membrane-potential responses is the same (Fig. 1C). This feature could allow neurons to differentially control the stimulus dependence 


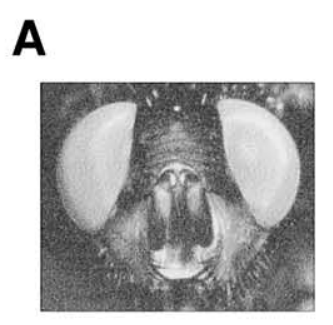

B

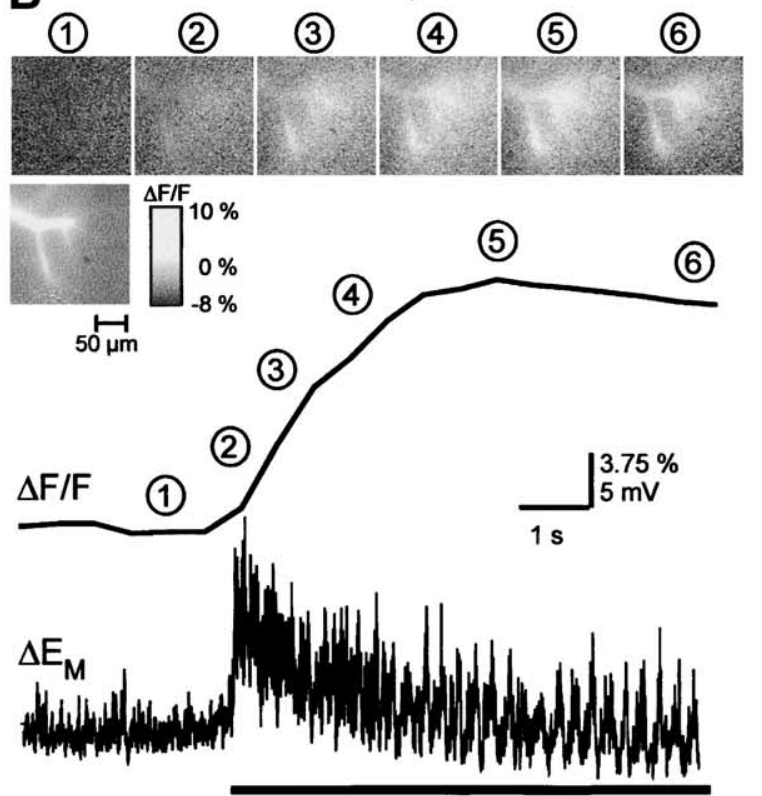

(6)

\section{(1)}
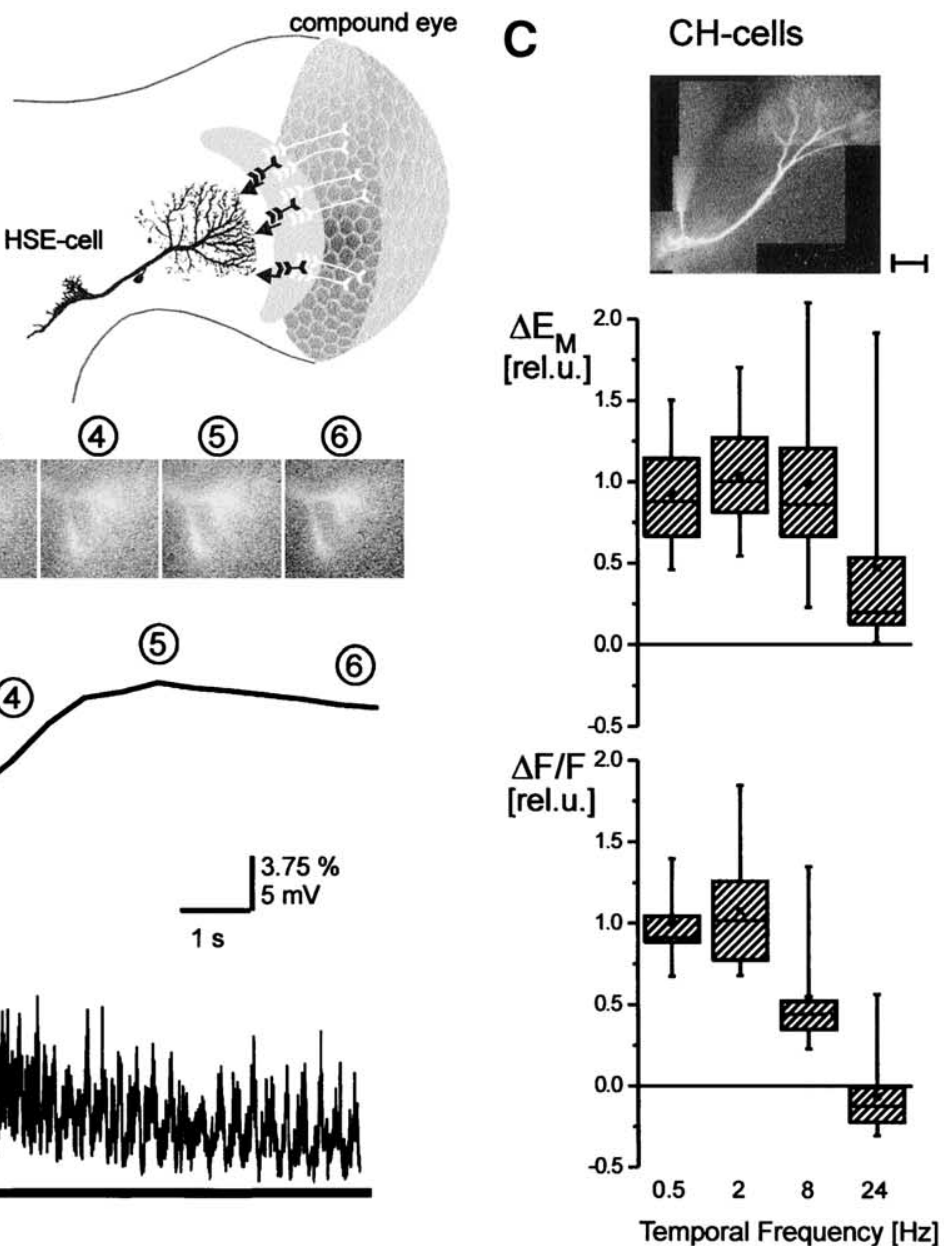

CH-cells

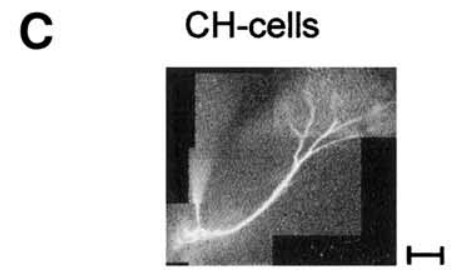

$\underset{\text { [rel.u. }}{\Delta \mathrm{E}_{\mathrm{M}}}$

HS-cells
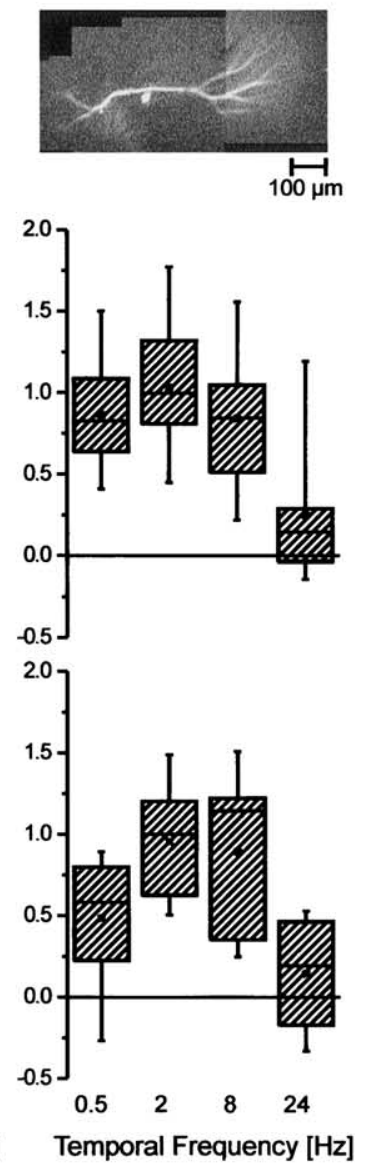

Fig. 1. Dendritic calcium signaling in visual motion-sensitive neurons of the fly. (A) (Left) head of a female blowfly (Calliphora vicina). Large compound eyes provide a nearly panoramic field of view. (Right) diagram of the visual motion pathway of the fly. Motion information is first calculated locally by a specific wiring of inputs from single ommatidia. Subsequently, the large dendrites of TCs retinotopically sample local estimates of motion in specific directions. The HSE-cell, which is shown here (reconstruction courtesy of K. Hausen), responds in a direction-selective manner to horizontal motion in the equatorial part of the visual field. (B) Changes in membrane potential and dendritic calcium concentration in an HS-cell measured in vivo during motion of a grating pattern with a temporal frequency of $4 \mathrm{~Hz}$ in the neuron's preferred direction. Calcium signals were detected by filling the neuron with a calcium-sensitive dye and measuring relative changes of the fluorescence intensity $(\Delta F / F)$ with a CCD camera. Calcium accumulation in the dendrite of the HS-cell is shown in a series of colorcoded pictures (raw fluorescence picture of the imaged area shown below and as a time-course of the signal spatially integrated across the dendrite (corresponding timing of the color-coded images indicated by the numbering). Pattern motion indicated by the horizontal bar. The membrane potential $\left(\Delta E_{M}\right)$ of the neuron, measured intracellularly in its axon, responds with a graded depolarization on which spike-like transients are superimposed. (C) Comparison of two classes of TCs, $\mathrm{CH}_{-}$, and HS-cells (individual members shown on top as reconstructions from fluorescence images), with respect to their membrane-potential responses and dendritic calcium responses. The two cell classes are assumed to perform different computational tasks, in the context of figureground discrimination in the case of $\mathrm{CH}$ - and of optomotor control in the case of HS-cells (105-109). $\mathrm{CH}$-cells possess dendritic output synapses 29. The dependence of membrane potential responses on motion velocity (given here as the pattern's temporal frequency) is similar for both cell classes (top left: data for $\mathrm{CH}$; top right: data for HS). In contrast, the magnitude of dendritic calcium responses differs significantly in its velocity dependence between the cell classes (bottom left: data for $\mathrm{CH}$; bottom right: data for $\mathrm{HS}$ ). All values are normalized to the response at $2 \mathrm{~Hz}$. Experimental data in part from ref. 28. 
A

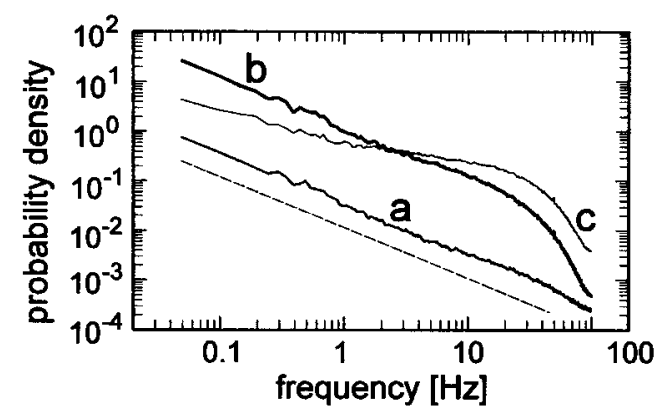

B
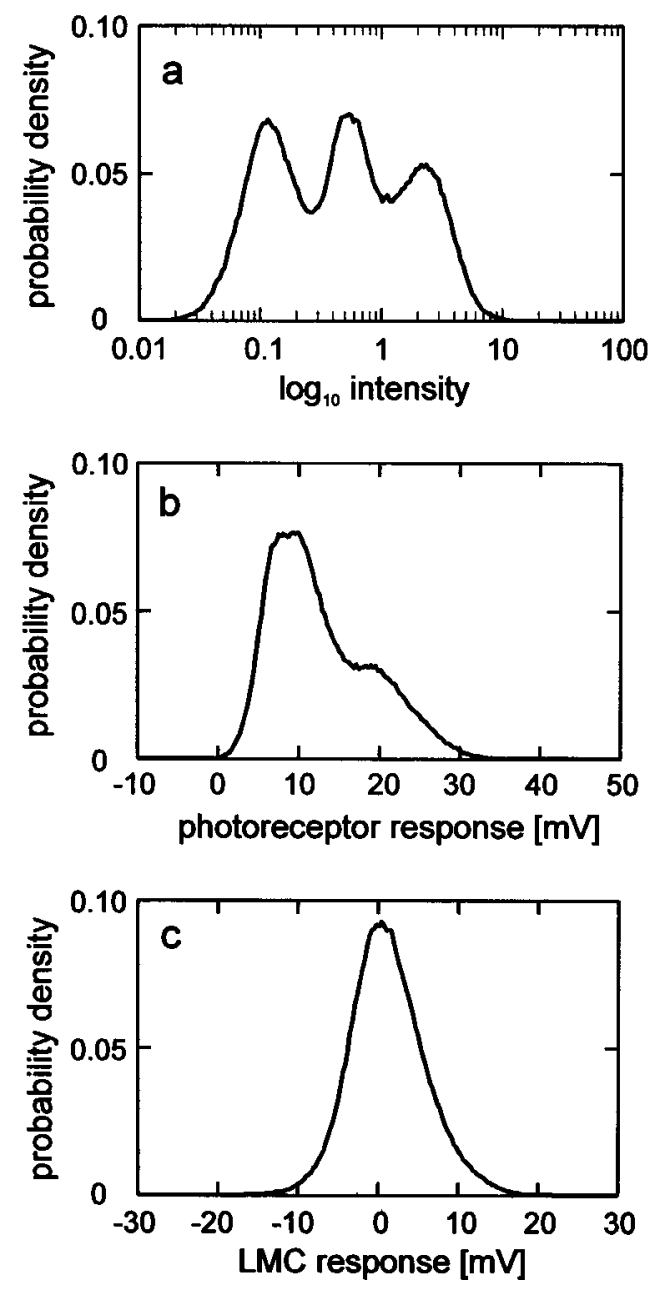

Fig. 2. Processing of natural times series of light intensities by the visual system of the fly. (A) Temporal power spectra of a 45-min stretch of intensity data, measured by a head-mounted device worn by a freely-walking person (a), and of the resulting of physiological processes, such as transmitter release or enzymatic activities, depending on whether the process is regulated by calcium, by the membrane potential, or by both.

In situ recording techniques have also been used in the fly to study how photoreceptors and their synaptic targets, the large monopolar cells (LMCs), process fluctuating light intensities, as encountered in natural situations (30). The fluctuations of light intensity through time ("time series") were behaviorally generated, not by monitoring the motions of a fly but by those of a human subject: a small optical device measuring light intensities was worn on a headband by a freely walking person. The measured time series of light intensities were subsequently presented to the fly during electrophysiological recordings. Power spectra of natural time series of intensities closely follow a $1 / \mathrm{f}$ relationship (with $\mathrm{f}=$ temporal frequency). These signals are low-pass filtered at the level of the photoreceptors. Further filtering is introduced by the LMCs. This processing of natural light intensities results in power spectra that are almost flat over a large range of temporal frequencies (Fig. 2A). Moreover, although the light intensities in time series of

responses in a photoreceptor (b) and an LMC (c) of the blowfly (Calliphora vicina). The photoreceptor's power spectrum closely follows a $1 / f$ relationship (with $f=$ temporal frequency), and its slope is shown by the dashed line. For $a$, power density is normalized to the average intensity, thus yielding a unit of (contrast) ${ }^{2} \mathrm{~Hz}^{-1}$. For $b$ and $c$, the units are $(\mathrm{mV})^{2} \mathrm{~Hz}^{1}$. (B) Probability density of the intensity measurement (a) and the resulting responses in a photoreceptor (b) and an LMC (c). Highly skewed intensity distributions are transformed into symmetrical distributions of photoreceptor and, even more, of LMC activity. Note that a roughly symmetrical distribution on the intensity data on a log scale (as in a) implies a very skewed distribution on a linear scale. The plots also indicate that photoreceptors and LMCs use a large part of their available response range when responding to natural stimuli. The units of the probability densities are $\left[\log _{10} \text { (intensity) }\right]^{-1}$ in $\mathbf{a}$, and $\mathrm{mV}^{-1}$ in $\mathbf{b}$ and c. Figures adapted from ref. (30). 
limited duration often have skewed probability density distributions, the response of photoreceptors and particularly that of LMCs is much more symmetrically distributed over the range of attainable membrane potential values (Fig. 2B). This shows that neurons at the first stages of the visual system utilize a large part of their available response range-thus efficiently exploiting the channel's information capacity - when they respond to natural stimuli. Along with linear filtering, fast gain control, and adaptation, contribute to this feature of sensory coding.

The filtering properties of photoreceptors are not uniform across various insect species. Differences in temporal filtering have been shown to match the demands imposed on photoreceptors by the behavioral repertoire of the animal (31). Fast-moving fly species possess rapidly responding photoreceptors, whereas slow-moving species exhibit more slowly responding ones. These differences in photoreceptor performance were shown to be the result of differences in the cells' equipment with delayed-rectifier potassium conductances (32).

The physiological mechanisms underlying the processing of sensory information have also been studied in orthopterans. Crickets are equipped with a mechanosensory system on antenna-like abdominal appendages known as cerci, which sense wind stimuli. The cerci enable the cricket to detect approaching predators in order to execute a rapid escape response. Sensory processing in this system exhibits several similarities to the processing of optic flow by TCs in the fly visual system. First, information from sensory afferents, in this case supplied by filiform hairs distributed on the cerci, is integrated by only a few giant interneurons that are located in the terminal abdominal ganglion (TAG) (33). Second, like the TCs, the interneurons in the TAG sample a large number of sensory inputs on their dendrites in a topographic fashion (34-36). Third, calcium accumulation appears to play a role in the dendritic computation of input information (37-40). What makes the study of informa- tion processing in the mechanosensory cercal system of the cricket attractive for the researches experimenter is that-similar to the fly visual system - electrophysiological recording and calcium imaging can be performed in vivo during sensory stimulation (Fig. 3A).

Calcium accumulation in the distal dendrite of the median giant interneuron (MGI) depends on the direction of air-puffs applied to the cerci (38). (Fig. 3B) In contrast, calcium accumulation in the "initial segment", a proximal dendritic region close to the axon of the MGI, and the spike response of the neuron, are not markedly dependent on direction. Nevertheless, distal dendritic calcium increase was suppressed when action-potential generation was blocked $(38,39)$. This is surprising because it indicates that dendritic calcium influx is critically dependent on the co-occurrence of spike backpropagation into the dendrite and synaptic input activity. What physiological functions can be attributed to calcium accumulation in the dendrites of the wind-sensitive interneurons in the TAG of the cricket? Recent evidence suggests that, in the MGI, dendritic calcium plays a role in the regulation of wind sensitivity via short-term depression of input synapses (Fig. 4). Activity-dependent changes of the excitatory postsynaptic potential (EPSP) slope indicative of synaptic depression were suppressed when calcium rises were blocked by injection of a calcium chelator into the MGI (40).

\section{Adaptation}

The range of intensities of sensory stimuli encountered by an animal in real life often encompasses several orders of magnitude. For instance, neurons in the visual system are confronted with changes in light intensity ranging from dim starlight to bright daylight. However, the neuronal signaling range, is limited by physiological properties such as the fixedreversal potential of membrane currents. The task of coding a large variety of input intensities by a limited bandwidth of output activities 


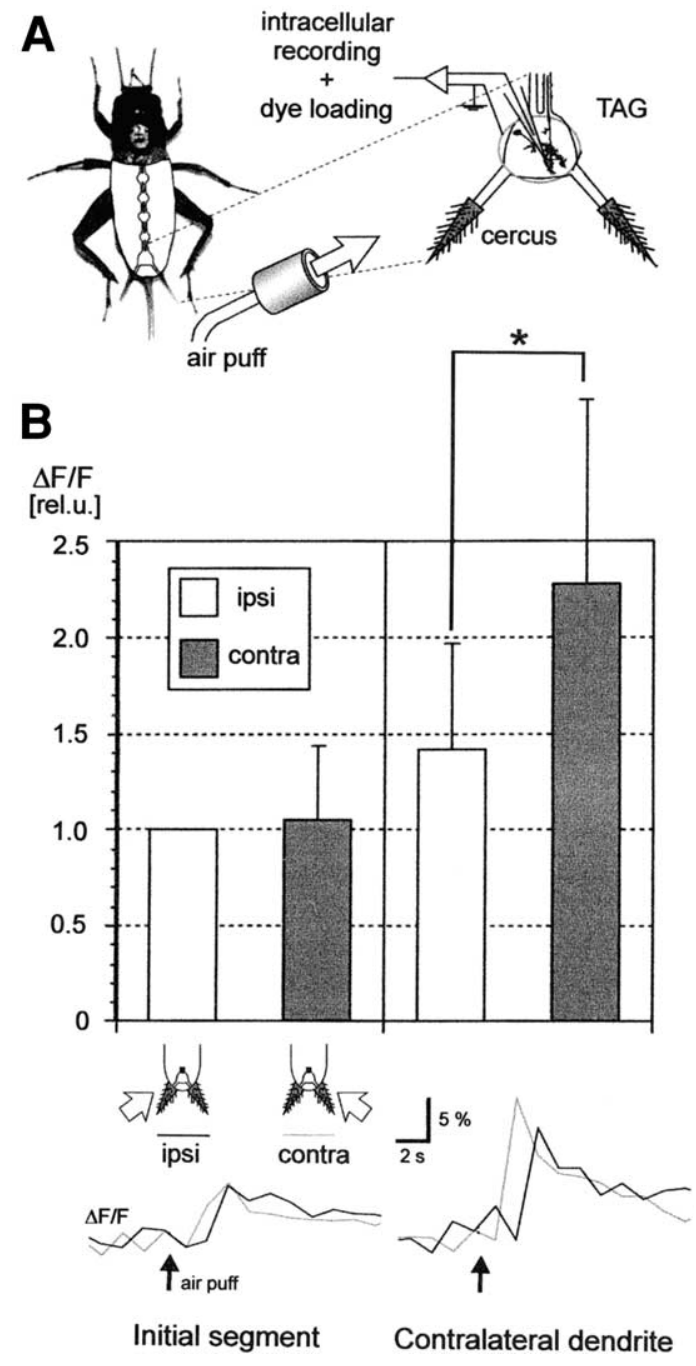

Fig. 3. Direction selectivity of wind-evoked calcium transients in interneurons of the cricket cercal system. (A) Diagram of the experimental set-up. An interneuron in the terminal abdominal ganglion (TAG) of the cricket (Gryllus bimaculatus) is recorded in vivo with an intracellular electrode and filled with a calcium-sensitive fluorescent dye. Air puffs are applied to the cercus located either ipsi- or contralateral to the neuron's soma. (B) Top, peak amplitudes of calcium concentration rises measured by confocal imaging of relative changes of the fluorescence intensity $(\Delta F / F)$ in response to air puffs to the ipsilateral (white columns) or the contralateral (shaded columns) cercus. Responses were measured either in the proximal "initial segment" of the dendrite (left two columns) or the distal contralateral dendrite (right two columns) of the neuron and nor- can only be solved by implementing physiological processes that temporarily shift the neuron's response range to the current prevailing stimulus intensity. In the fly, photoreceptor adaptation can be studied in vivo by penetrating a single photoreceptor in the intact animal with a recording electrode. Photoreceptor activity has been recorded in response to light flashes of variable intensity against background illumination (41-44), and in response to more naturalistic luminance fluctuations (45-48). Properties of the phototransduction cascade and the photoreceptor membrane take part in the process of adaptation. At low-background-light intensities, fly photoreceptors generate discrete elementary responses (e.g., quantum bumps) to single photons. With increasing light intensity, quantum bumps become faster and smaller, and are integrated into larger and faster voltage responses that more accurately follow the time-course of the light stimulus. It has been shown that voltagesensitive potassium conductances adjust the properties of the photoreceptor membrane to the changes in gain and dynamics of the phototransduction cascade that are associated with different light conditions (49). Recently, stable in vivo recordings from photoreceptors proved to be feasible in large flies such as Calliphora as well as in Drosophila $(47,48)$. This genetically accessible animal model will offer the opportunity to study how molecular processes lead to basic neuronal functions such as adaptation in a behavioral context, and how these are compromised by malfunctions in individual steps of the signaling machinery.

malized to the response in the initial segment to ipsilateral stimulation. Below, sample time-courses of $\Delta \mathrm{F} / \mathrm{F}$ in the initial segment (left) and the contralateral dendrite (right) upon stimulation of the ipsilateral (thick line) or contralateral (thin line) cercus. Calcium accumulation in the contralateral dendrite was direction selective, yielding larger response amplitudes during stimulation of the contralateral cercus (asterisk indicates $p<0.05$, t-test). Figures adapted from ref. $(38,40)$. 


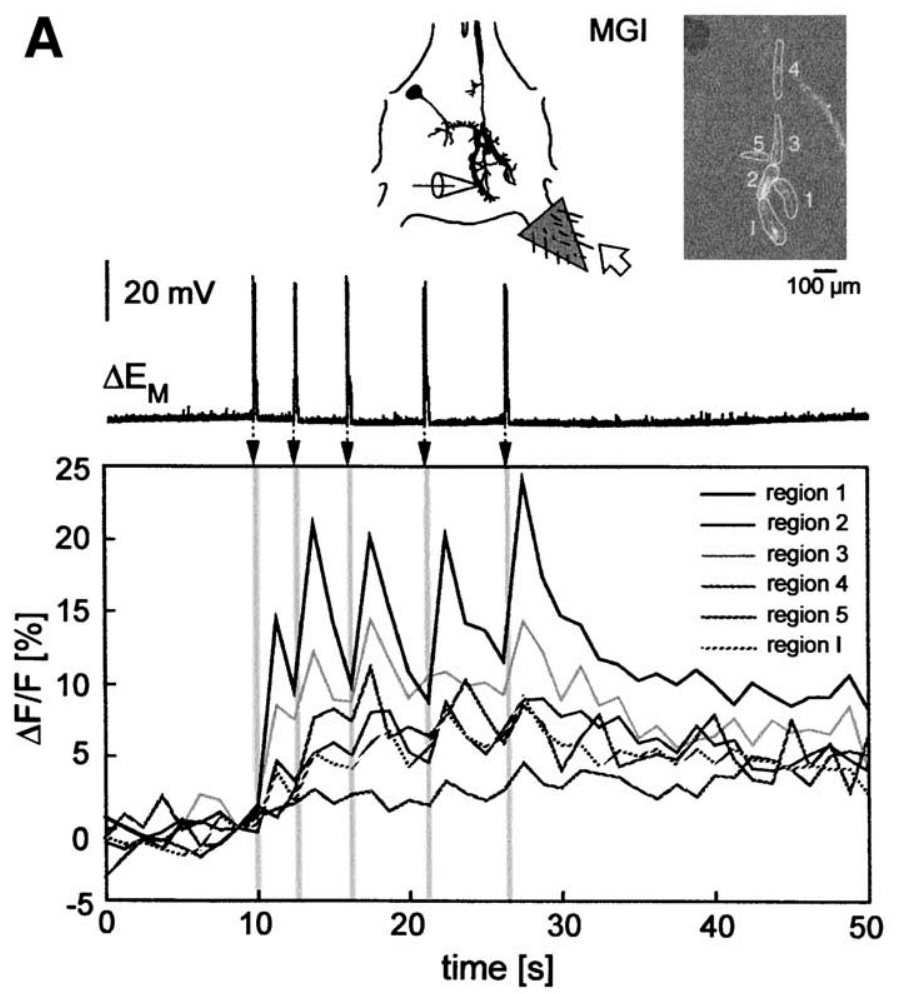

B

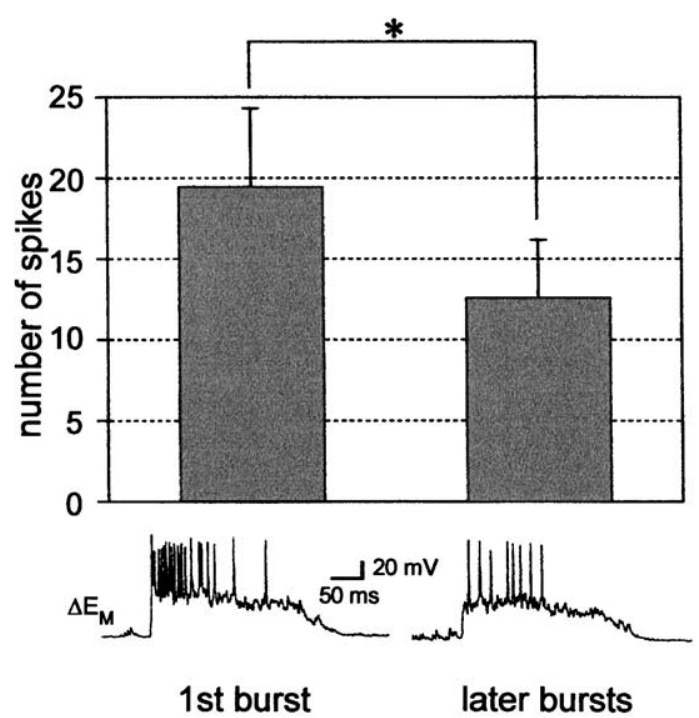

Fig. 4. Calcium accumulation and regulation of wind-sensitivity by short-term depression in the cricket cercal system. (A) Membrane potential changes $\left(\Delta E_{M}\right)$ and relative calcium concentration changes $(\Delta F / F)$ in the $\mathrm{MGl}$ of the cricket cercal system are measured in vivo during repetitive air puff stimulation. Calcium accumulation was determined for various regions of the neuron (see inset) and was largest in the dendritic branch on the stimulated side (region 1). Region 1 indicates the neurite where the intracellular electrode was inserted. (B) Attenuation of wind-evoked responses during successive air-puff stimulation. Spike number in response to the first air puff is signicantly higher than that to subsequent ones (asterisk indicates $p<0.005$, t-test). Time-courses of sample responses are shown below. Response attenuation is caused by a reduction of the EPSP slope and can be blocked by injection of BAPTA into the MGI (data not shown), suggesting calcium-dependent short-term depression. Figures adapted from ref. (40).

Intracellular calcium dynamics can play a behaviorally relevant role in the regulation of neuronal sensitivity. This was demonstrated in a seminal study on the cricket auditory system (50). The response of the omega neuron, which is involved in phonotaxis during mating behavior, shows a so-called forward-masking effect when stimulated with simulated cricket calling songs: A loud sound suppresses the response to subsequent sounds. Optical recording of calcium dynamics in vivo revealed that calcium accumulates in the omega neuron during sensory stimulation. This calcium accumulation is associated with an attenuated response to repetitive stimulation, and with a hyperpolarization of the omega neuron following the cessation of a stimulus. The hyperpolarizing after-response is likely to be caused by a calcium-dependent ionic conductancee.g., calcium-activated potassium channels, which are known to regulate excitability in many neurons (8). Two findings provide further evidence for a regulation of sensitivity by a calcium-dependent conductance in the omega neuron (50): Neuronal excitability is increased by injection of high concentrations 
of a calcium buffer and decreased by photolytic release of calcium from a caged calcium compound. The latter manipulation also resulted in a hyperpolarization when applied to the omega neuron during a period of rest.

Adaptation also has a functional relevance in the visual motion pathway of the fly. Largefield motion-sensitive neurons in the fly brain - the TCs-adapt to ongoing stimulation by decreasing their response magnitude (51). At the same time, their sensitivity to changes in pattern velocity seems to be preserved $(51,52)$. However, as shown by recent studies, adaptation in the visual motion system of the fly is a composite phenomenon, with several processes functioning either in sequence or in parallel (53-55). Moreover, studies have recently questioned whether adaptation induces a shift of the velocity-response curve to the range of prevailing stimulus velocities (56). In earlier studies, such a shift was concluded to be a key function of motion adaption $(51,57,58)$. All this makes it difficult to interpret the impact of adaptation on neuronal coding and on the behavioral output of the system.

One component of adaptation can be distinguished by its direction selectivity. It is prominent only during motion in the preferred direction, but not when the direction of motion is reversed (the null direction, which hyperpolarizes graded-potential TCs) or perpendicular to the preferred direction (which causes adaptation although no voltage response is elicited during motion [see ref. 54]). Interestingly, the physiological mechanisms that occur during preferred-direction adaptation may be very similar to those found in the cricket omega neuron. As in the omega neuron, a hyperpolarizing after-response follows the cessation of excitatory stimuli, such as preferred-direction motion in the case of fly TCs (55). The time-courses of the after-response and of the recovery from adaptation closely resemble each other, both in the cricket omega neuron and in fly TCs $(50,55)$. During preferred-direction motion, calcium accumulates in the dendrites of TCs. Motion input on TC dendrites is retinotopically organized, and calcium elevations remain locally restricted to activated portions of the dendrite $(59,60)$. Calcium-dependent mechanisms underlying motion adaptation could thus account for the finding that adaptation affects only those parts of the receptive field in which the adapting stimulus has been presented, instead of spreading out across the entire receptive field (51). Correlations between the magnitude of dendritic calcium accumulation and the amplitude of the hyperpolarizing afterresponse in two classes of TCs provide evidence for the regulation of motion adaptation by calcium (Fig. 5).

The natural input of a sensory system is characterized by its strength, and also by how much and how rapidly input strength fluctuates over time. The dynamic properties of sensory stimuli may differ considerably, depending on the sensory modality and the behavioral context. For instance, input to the visual motion system of flies is expected to differ in its statistics during walking and during flight. The degree to which motion adaptation in a TC is dependent on the statistical parameters of the stimulus has been investigated in two recent studies $(53,61)$. Motion stimuli with velocity fluctuations characterized by different variance were presented. One study concluded that the input-output relation of the neuron is stretched or compressed in order to match a broad or a narrow distribution of input signals, respectively (61). Such an "adaptive rescaling" would help to maximize information transmission. However, adaptation implies that neuronal activity depends on the context. Thus, a particular activity level does not correspond unambiguously to a certain stimulus strength. In the second study, it was concluded that this ambiguity can be resolved by adaptation on multiple time-scales and by representation of various stimulus properties by different parameters of spike train statistics (53).

\section{Synaptic Information Transfer}

The usefulness of biologically relevant patterns of stimulation also applies to the investi- 

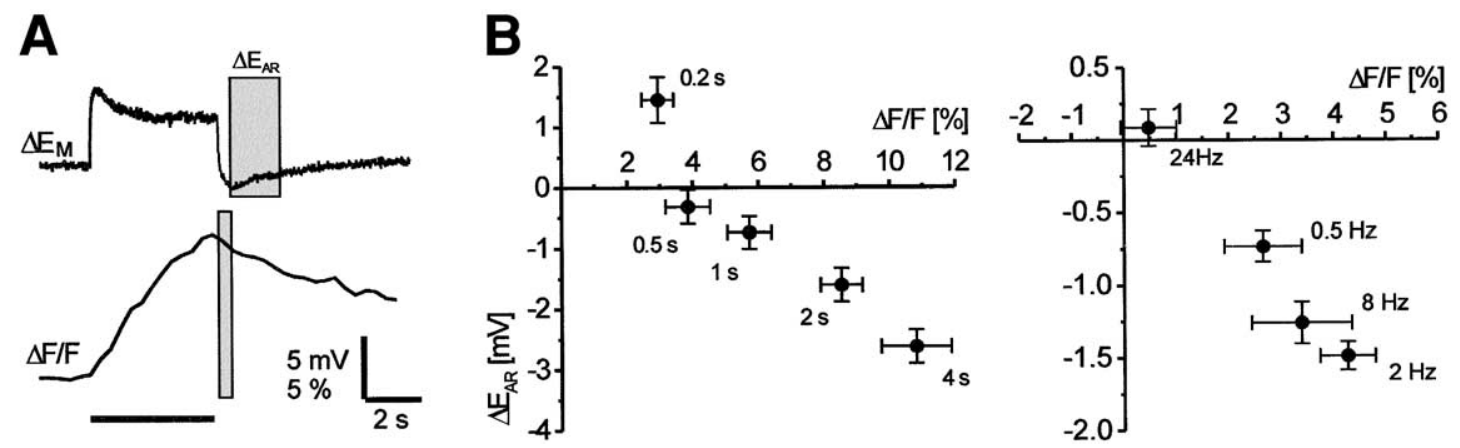

Fig. 5. Role of dendritic calcium in direction-selective adaptation of visual motion-sensitive neurons. (A) Membrane potential responses (upper trace, averaged response, $n=15$ cells) and calcium accumulation in the dendrite (lower trace, averaged response, $n=5$ cells) of fly HS-neurons were measured during presentation of preferred-direction motion stimuli. A hyperpolarizing after-response is prominent upon the cessation of motion. Responses to motion stimuli are attenuated while the neuron is in its hyperpolarized state (55). To analyze whether the after-response could be caused by a calcium-dependent mechanism, the relationship between the magnitude of the after-response $\left(\Delta E_{A R}\right.$, shaded box) and the amount of calcium accumulated at the end of the stimulus (shaded box) were determined. (B) Left, correlation between the magnitude of the after-response $\left(\Delta E_{A R}\right)$ and dendritic calcium accumulation $(\Delta F / F)$ as determined for motion stimuli of variable duration (given beside each data point). Data points show mean $+/-$ SE $(n=5-16$ cells). A correlation between after-responses and dendritic calcium in HS-neurons is also seen when stimuli of variable velocity (given as temporal frequency beside each data point) are applied (right). Data points show mean $+/-\mathrm{SE}(n=9-30$ cells). Experimental data from ref. 55.

gation of synaptic performance, as underscored by a recent study on midbrain electrosensory neurons in Eigenmannia (62). Both sensory stimulation and artificial stimulation of afferents at naturalistic pulse rates elicit short-term synaptic depression in the electrosensory pathway. By attenuating high temporal frequencies of the stimulus more than low ones, short-term synaptic plasticity acts as a temporal filter. However, plasticity-induced filtering differs from low-pass filters such as those that result from passive electrical properties of neurons in one important aspectunlike an ordinary low-pass filter, filtering by short-term depression leaves the onsets of high-temporal frequency stimuli unattenuated. This feature may be highly relevant for the fish in behavioral situations, since in this way the neuron preserves its ability to detect sensory transients (63).

Transfer and processing of sensory information at synapses has also been studied in the visual system of the fly. Conclusions about the natural performance of the first synapse in the system, the synapse between photoreceptors and LMCs, can be drawn by recording the responses of both types of neurons to light stimuli that fluctuate dynamically in their intensity (64). By superimposing these stimuli on a wide range of background intensities, it is possible to investigate how the system copes with the large spectrum of illumination conditions encountered during locomotion in an inhomogeneous habitat and during the course of a day. Both photoreceptors and LMCs shift their membrane potentials in a graded way instead of generating action potentials (65). Thus, there is graded and continuous transmitter release at the photoreceptor-LMC synapse, as well as at the synapse between photoreceptors and bipolar cells in the vertebrate retina (66-68). Since it is not feasible to record photoreceptors and LMCs simultaneously, responses of photoreceptors and LMCs elicited by identical stimuli and recorded successively were compared. The interposed synapse was 

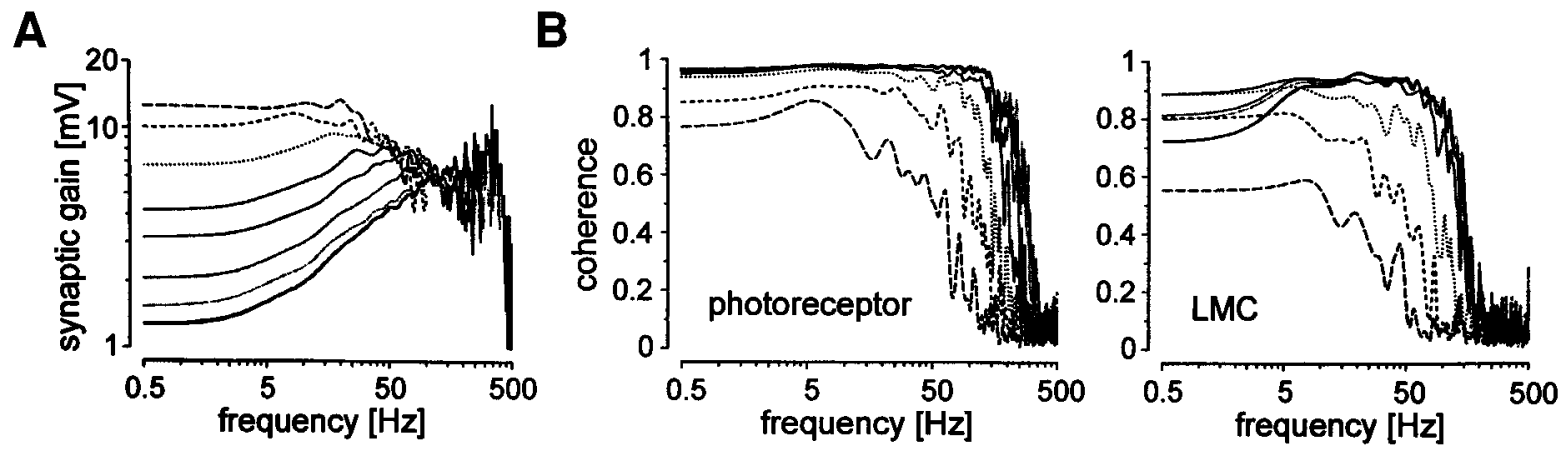

Fig. 6. Transfer characteristics of photoreceptor-interneuron synapses. (A) Voltage responses of photoreceptors and their postsynaptic targets, LMCs, to pseudorandomly modulated contrast stimuli were recorded at various adapting background intensities. Synaptic gain is calculated and plotted as a function of modulation frequency and background luminance (line styles; solid line, highest background intensity). In the low-frequency range, synaptic gain is about ten times higher at dim backgrounds compared to bright backgrounds. Moreover, the region of highest synaptic gain shifts from the low- to the high-frequency range with increasing background intensity. (B) Relationship between visual stimulus and neuronal response expressed by the coherence function for photoreceptors (left) and LMCs (right). In a linear and noise-free system, the coherence value is one. For high background intensities, coherence values close to one are reached by photoreceptors in the frequency range between 0.5 and $150 \mathrm{~Hz}$, and by LMCs between 5 and $150 \mathrm{~Hz}$. This is consistent with linear synaptic transmission from photoreceptors to LMCs in the frequency-range between 5 and $150 \mathrm{~Hz}$ under light-adapted conditions. Figures adapted from ref. 64.

concluded to adjust its transfer characteristics according to the demands imposed by different background light intensities (64). In dim light, overall responses of the photoreceptors aredespite adaptation-rather small. Moreover, they are fairly slow. Thus, in the high-frequency range, there is not much signal, but all the more noise, which is partly the result of discrete, highly amplified quantum bumps. To meet the requirements of dim light conditions, overall synaptic gain is high, but the synapse attenuates high input frequencies (Fig. 6A). With increasing mean light level, both the overall amplitude of the photoreceptor response and its signal-to-noise ratio in the high-frequency range become larger. Synaptic transfer adapts to this situation by a decrease in gain, mainly in the low-frequency range. In this way, the postsynaptic response is prevented from saturating, and begins to favor high frequencies over low ones.

The coherence function can test to what extent a neural response to a temporally fluctuating stimulus can be accounted for by a sys- tem operating in a linear and reliable way. An absolutely linear and noise-free performance results in a coherence value of one (Fig. 6B). Under light-adapted conditions, the coherence for both photoreceptors and LMCs comes close to one in the frequency range from $5-150 \mathrm{~Hz}$. This is consistent with a remarkably linear performance of photoreceptor-LMC synapses over a large range of frequencies under lightadapted conditions (64).

Linearity of synaptic information transfer over a wide range of operating conditions also seems to characterize another synapse in the fly visual system (69): VS-neurons, one class of large-field motion-sensitive TCs, synapse on another TC, the V1-neuron. VS-neurons integrate retinotopically arranged dendritic input into presynaptic output, which consists of graded membrane-potential shifts on which spike-like depolarizations are superimposed (70-72; see Fig. 7A). V1 receives excitatory input from three VS-neurons, transforms this information into spike-trains, and transmits it to the contralateral brain hemisphere. Single 


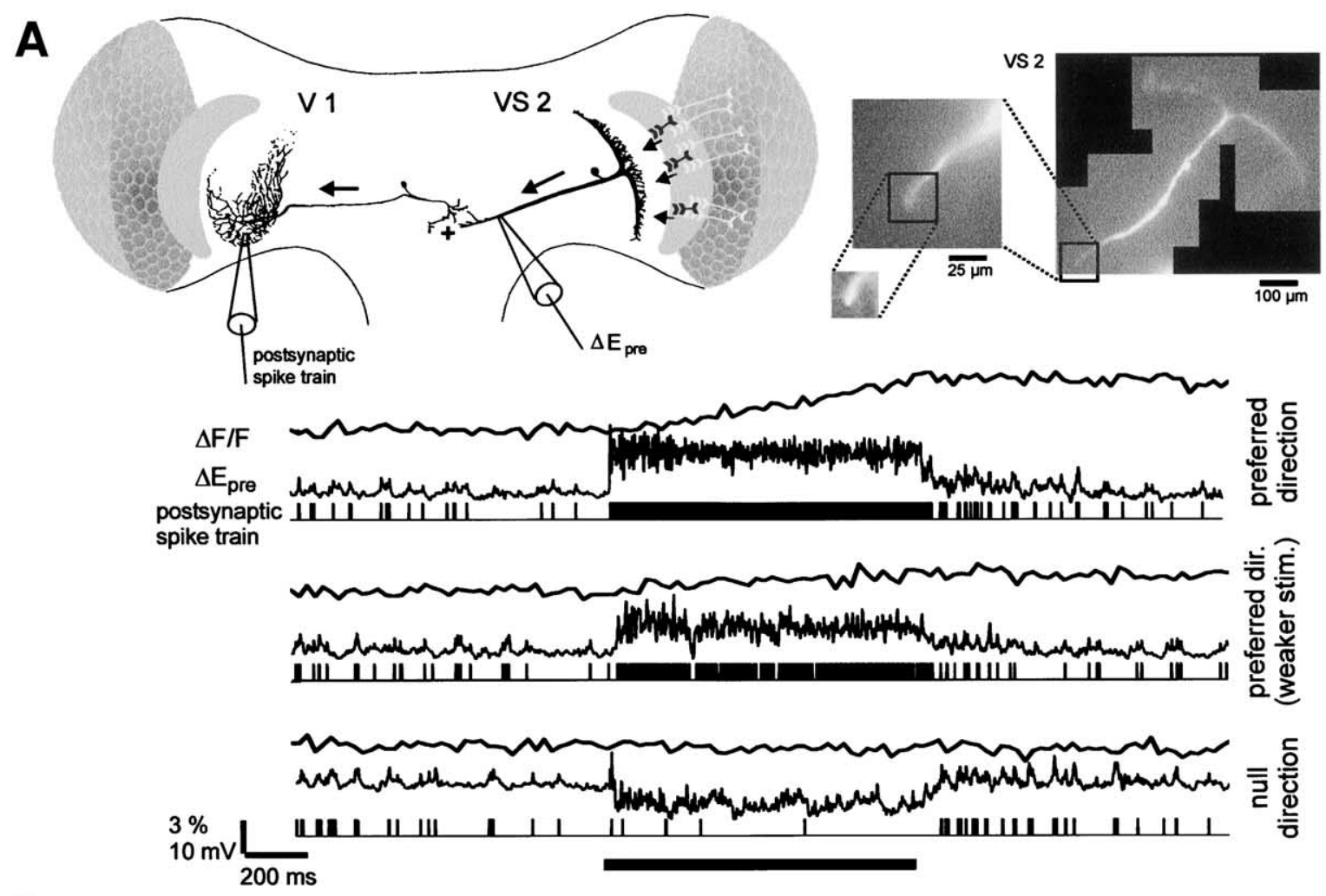

B
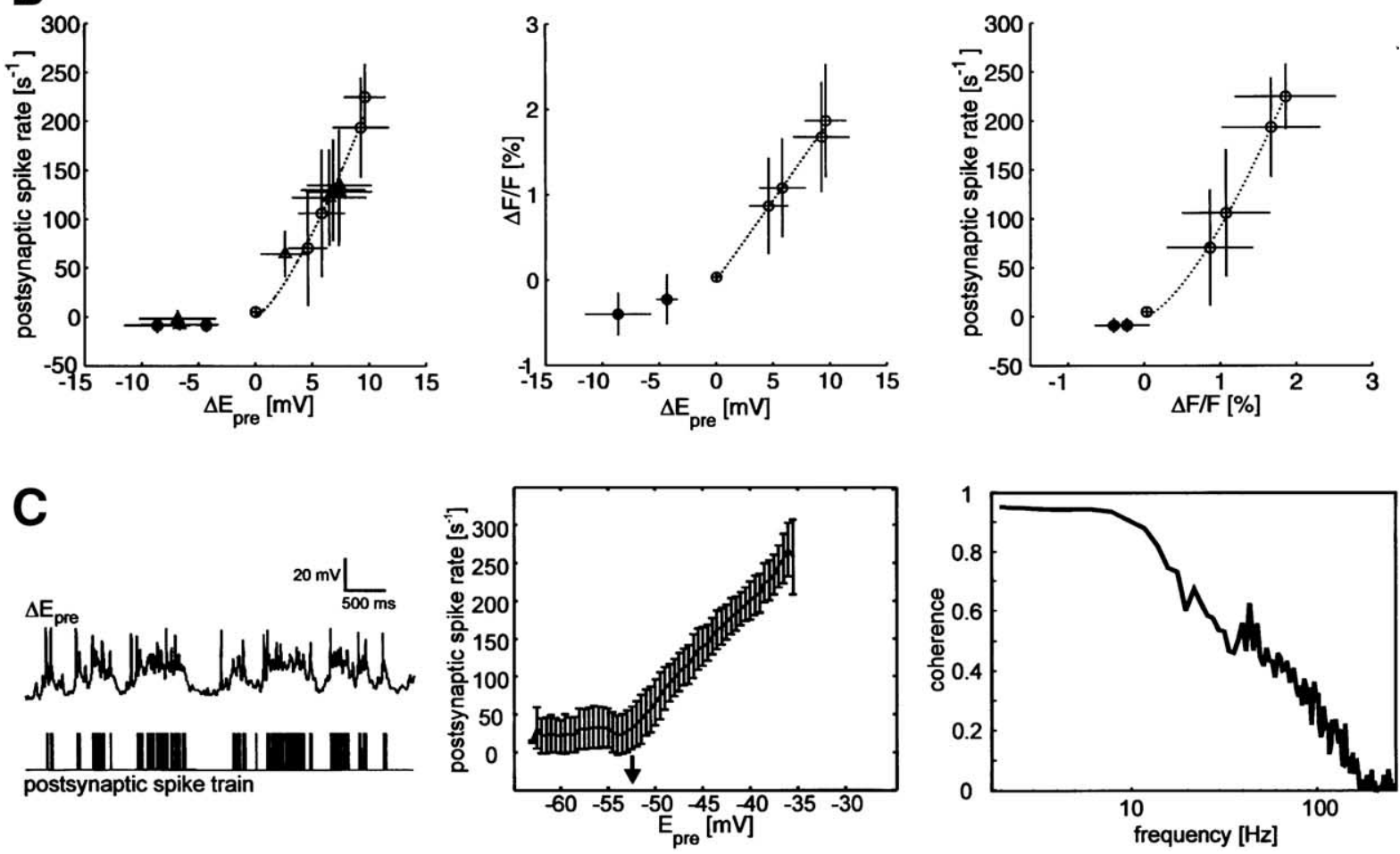
Fig. 7. Synaptic performance in the natural operating range. (A) In the visual system of the blowfly (Calliphora vicina), graded-potential VS-neurons respond to vertical motion in a large portion of the ipsilateral visual field. The spiking V1-neuron receives excitatory synaptic input from three VS-neurons, among them VS 2, and transmits this motion information to the contralateral brain hemisphere (see diagram upper left, cell reconstructions reproduced from refs. 72,75). During visual stimulation of the presynaptic VS-neurons, the membrane potential of a VS-neuron $\left(\Delta E_{\text {pre }}\right)$ was intracellularly recorded, and the postsynaptic spike train was monitored by an extracellular electrode in $\mathrm{V} 1$ 's output arborization. The VS-neuron was filled with a calcium-sensitive dye (see raw fluorescence images, upper right) in order to measure calcium concentration changes in the presynaptic region by fluorescence imaging with a CCD-camera. The time-courses show presynaptic calcium concentration $(\Delta F / F)$ and membrane potential $\left(\Delta E_{\text {pre }}\right)$, and postsynaptic spike trains (action potentials indicated by upright lines). All three were measured simultaneously during preferred-direction motion of two strengths, which was varied by changing brightness and contrast of the motion pattern (upper and middle traces) and during nulldirection motion (lower traces). (B) Relationships between pre- and postsynaptic responses (mean $+/-\mathrm{SD}, n=$ 4-11 cell pairs) evoked by visual motion stimuli of different strengths. The relationships between presynaptic depolarizations, presynaptic calcium accumulation and postsynaptic spike rates are, on average, linear over the entire range of stimulus strengths (open symbols). Rectification is present during null-direction motion, because it elicits marked presynaptic hyperpolarization but only weak changes in presynaptic calcium and in postsynaptic spike rates (solid symbols). Values of zero correspond to resting levels. For preferred-direction motion, the relationships were fitted by power-law functions of the type $y=a x^{b}$ (dotted lines, $b$ values from left to right: 1.4, $1.0,1.4)$. (C) Synaptic transfer of dynamical motion information. Left, presynaptic membrane potential $\left(\Delta E_{\text {pre }}\right)$ and postsynaptic spike-train during presentation of white-noise velocity fluctuations. Middle, relationship between instantaneous spike rate (estimated from the spike train as the inverse of the interspike interval) and $\Delta \mathrm{E}_{\text {pre }}$ (both temporally filtered by a Gaussian with $\sigma=20 \mathrm{~ms}$ ). The arrow indicates the resting membrane potential of the VS-neuron. Right, the coherence function for the transfer of the presynaptic membrane potential into the postsynaptic spike-train (values below the resting potential were set to zero to account for the rectification during ND motion). A coherence value of one is consistent with a linear and totally noise-free system. Experimental data from refs. $(69,73)$.

VS-neurons and the V1-neuron were recorded simultaneously in vivo. Synaptic performance was tested in its naturally relevant activity range by presenting visual motion stimuli of various strengths. Over the entire range, presynaptic depolarizations were found to be linearly transformed into postsynaptic spike activity (Fig. 7B).

Recently, information transfer at VS-V1 synapses was also investigated during presentation of motion stimuli that fluctuated continually in their velocity, as is typical for optic flow during locomotion (73). Even under this highly dynamic condition, synaptic transfer complies with linear and reliable system properties for presynaptic membrane-potential fluctuations up to approximately $10 \mathrm{~Hz}$ (Fig. 7C). This corresponds to the dynamic range in which most motion signals are encoded in the visual system (74). Higher frequencies are increasingly dominated by noise. Synaptic transfer characteristics may thus be matched to the temporal limitations of visual motion processing. Nevertheless, information on a millisecond time-scale can also be transferred via VS-V1 synapses, although by nonlinear mechanisms. These promote a tight temporal coupling of postsynaptic spikes to presynaptic spike-like depolarizations (73).

Fly TCs are involved in the optomotor control of the flight course, which relies on the comparison of motion information from both eyes (75). Linearity of information transfer between TCs may be advantageous in this context, since it guarantees a consistent dependency of neuronal responses on stimulus parameters such as motion velocity, regardless of the number of intervening synapses. However, it seems difficult to accomplish linearity over a large activity range when considering 
the often highly nonlinear physiological mechanisms underlying synaptic transmission. For instance, such processes include the activation characteristics of presynaptic calcium channels and the calcium-dependency of vesicle exocytosis. The latter has been found at many synapses to be regulated by calcium in a cooperative way, consistent with the finding that binding of more than one calcium molecule to the molecular sensors of the release machinery is necessary to trigger exocytosis (76). Hill coefficients of 3 or 4 seem to characterize calcium cooperativity of transmitter release at most analyzed synapses (1,77-82). However, at VS-V1 synapses, both the relationship between membrane depolarization and calcium accumulation in the presynaptic region and that between the latter and the postsynaptic spike response were found to be fairly linear (69) (Fig. 7B).

Two considerations can help to explain these results: i) Synaptic signaling in the fly visual system may have been shaped by the specific computational demands in the context of optomotor behavior. ii) General differences appear to exist between spike-mediated and graded synaptic transmission. Presynaptic calcium channels in graded-potential neurons often have a lower activation threshold compared to the ones in spiking neurons $(65,83)$. Obviously, this also applies to the calcium channels in TCs $(26,84)$. Furthermore, the resting membrane potential of graded-potential neurons is often not as negative as that of spiking ones (65). Thus, during synaptic activity, presynaptic membrane-potential values are modulated around the midpoint of the voltage-response curve of the calcium channels.

In regard to calcium cooperativity of transmitter release at graded synapses there is evidence for either relatively linear dependency of release on presynaptic calcium $(83,85)$, or for co-existence of multiple components of transmitter release, which deviate in their time-course and potentially also in their calcium dependency (86-88). There is also evidence for molecular differences in the transmitter-release machinery between spik- ing and graded-potential synapses: the isoform expression of syntaxin, which is critical for calcium cooperativity of release (89), is different in graded ribbon synapses of the vertebrate retina compared to spike-operated synapses $(90,91)$. Specializations that allow graded synapses to operate linearly may be advantageous from an information-theoretical point of view. Whereas steep supralinearities during synaptic transmission could help to isolate all-or-none signals, such as actionpotentials from membrane-potential noise, such nonlinearities would degrade the transfer of small amplitude signals at graded synapses (see Fig. 8).

\section{Conclusions and Perspectives}

This review outlines how some of the approaches and techniques that have been successfully applied for a long time to in vitro preparations are beginning to contribute to in vivo investigations of neuronal functioning. Furthermore, new experimental procedures, specifically designed to elicit neuronal activity resembling what occurs during the animal's natural behavior as much as possible, are implemented in an increasing number studies. For instance, visual stimuli experienced by animals during locomotion or movement of their eyes can be reconstructed when either the animal's natural behavioral sequences or its reactions in closed-loop experiments are monitored. These behaviorally generated stimuli can subsequently be replayed during neuronal recording and/or used for modeling of neuronal responses $(92,93)$. In the fly, this technique has already been used successfully for optic flow during unrestrained walking (94-96) and during tethered flight $(25,97)$. Next, it will also be implemented for free flight, since methods are now available both for high-resolution recording of flight trajectories (98-101) and for presenting panoramic visual stimuli at sufficiently rapid rates (102).

In larger animals, miniaturized recording equipment even allows the researcher to 


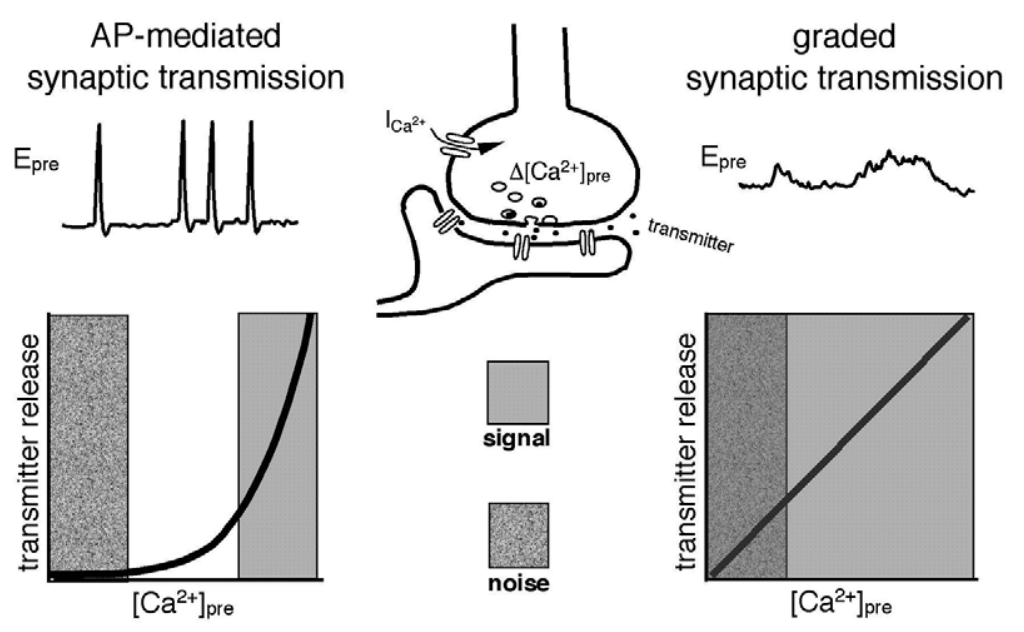

Fig. 8. Diagram illustrating hypothetical differences between modes of synaptic transmission. The transfer of action potentials, (e.g., large-amplitude all-or-none signals), would benefit from a supralinear relationship between transmitter release and presynaptic calcium concentration, $\left[\mathrm{Ca}^{2+}\right]_{\mathrm{pre}}$, (or in any other synaptic mechanism), yielding an increase in signal-to-noise ratio (left). At a graded synapse, in contrast, there may be signals of both large and small amplitude. Transfer of the latter would be degraded by synaptic supralinearities, but would be preserved by linear transfer characteristics (right).

record from neurons in the brain of freely moving animals (see refs. 92,103). Most recently, a technique for fluorescence imaging of calcium concentrations in the cortical neurons of freely moving rats has been introduced (104). Such approaches will help to reveal how cellular processes are organized to endow the nervous system with the ability to reliably perceive stimuli and properly execute behavioral tasks in a complex and continually changing environment.

\section{Acknowledgment}

The work done in our laboratory was supported by the Deutsche Forschungsgemeinschaft (DFG). We are grateful to Mikko Juusola, Hans van Hateren, and Hiroto Ogawa for kindly providing figures for this manuscript. We also thank Jack Waters and two anonymous reviewers for their helpful comments on earlier versions of this manuscript.

\section{References}

1. Dodge F. A., Jr. and Rahamimoff R. (1967) Cooperative action of calcium ions in transmitter release at the neuromuscular junction. J. Physiol. 193, 419-432.

2. Fatt P. and Katz B. (1951) An analysis of the end-plate potential recorded with an intra-cellular electrode. J. Physiol. 115, 320-370.

3. Thaler C., Li W., and Brehm P. (2001) Calcium channel isoforms underlying synaptic transmission at embryonic Xenopus neuromuscular junctions. J. Neurosci. 21, 412-422.

4. Yau K. W. and Nakatani K. (1985) Lightinduced reduction of cytoplasmic free calcium in retinal rod outer segment. Nature 313, 579-582.

5. Nakatani K. and Yau K. W. (1988) Calcium and light adaptation in retinal rods and cones. Nature 334, 69-71.

6. Matthews H. R. and Fain G. L. (2001) A lightdependent increase in free $\mathrm{Ca} 2+$ concentration in the salamander rod outer segment. J. Physiol. 532, 305-321. 
7. Jentsch T. J. (2000) Neuronal KCNQ potassium channels: physiology and role in disease. Nat. Rev. Neurosci. 1, 21-30.

8. Sah P. (1996) $\mathrm{Ca}(2+)$-activated K+ currents in neurones: types, physiological roles and modulation. Trends Neurosci. 19, 150-154.

9. Wu L. G., Westenbroek R. E., Borst J. G., Catterall W. A., and Sakmann B. (1999) Calcium channel types with distinct presynaptic localization couple differentially to transmitter release in single calyx-type synapses. J. Neurosci. 19, 726-736.

10. Turner R. W., Maler L., and Burrows M. (1999) Electroreception and electrocommunication. J. Exp. Biol. 202, 1167-1458.

11. Heiligenberg W. (1976) Electrolocation and jamming avoidance in the mormyrid fish Brienomyrus. J. Comp. Physiol. [A] 109, 357-372.

12. Westby G. W. M. (1974) Assessment of the signal value or certain discharge patterns in the electric fish, Gymnotus carapo by means of playback. J. Comp. Physiol. [A] 92, 327-341.

13. Knudsen E. (1974) Behavioral thresholds to electric signals in high frequency electric fish. J. Comp. Physiol. [A] 91, 333-353.

14. Rose G. J. and Fortune E. S. (1996) New techniques for making whole-cell recordings from CNS neurons in vivo. Neurosci. Res. 26, 89-94.

15. Fortune E. S. and Rose G. J. (1997) Passive and active membrane properties contribute to the temporal filtering properties of midbrain neurons in vivo. J. Neurosci. 17, 3815-3825.

16. Haag J. and Borst A. (1996) Amplification of high-frequency synaptic inputs by active dendritic membrane processes. Nature 379, 639-641.

17. Hausen K. (1984) The lobula-complex of the fly: structure, function and significance in visual behaviour, in Photoreception and vision in invertebrates (Ali M. A., ed.), Plenum Press, New York, London, pp. 523-559.

18. Egelhaaf M. and Borst A. (1993) A look into the cockpit of the fly: visual orientation, algorithms, and identified neurons. J. Neurosci. 13, 4563-4574.

19. Egelhaaf M., Kern R., Krapp H. G., Kretzberg J., Kurtz R., and Warzecha A.-K. (2002) Neural encoding of behaviourally relevant visualmotion information in the fly. Trends Neurosci. 25, 96-102.

20. Hausen K. (1982) Motion sensitive interneurons in the optomotor system of the fly: II. The horizontal cells: receptive field organization and response characteristics. Biol. Cybern. 46, 67-79.

21. Hausen K. (1982) Motion sensitive interneurons in the optomotor system of the fly: I. The horizontal cells: structure and signals. Biol. Cybern. 45, 143-156.

22. Krapp H. G. and Hengstenberg R. (1996) Estimation of self-motion by optic flow processing in single visual interneurons. Nature 384, 463-466.

23. Egelhaaf M. (1985) On the neuronal basis of figure-ground discrimination by relative motion in the visual system of the fly: III. Possible input circuitries and behavioural significance of the FD-cells. Biol. Cybern. 52, 267-280.

24. Egelhaaf M. (1985) On the neuronal basis of figure-ground discrimination by relative motion in the visual system of the fly: II. Figure-detection cells, a new class of visual interneurons. Biol. Cybern. 52, 195-209.

25. Kimmerle B. and Egelhaaf M. (2000) Performance of fly visual interneurons during object fixation. J. Neurosci. 20, 6256-6266.

26. Haag J., Theunissen F., and Borst A. (1997) The intrinsic electrophysiological characteristics of fly lobula plate tangential cells: II. Active membrane properties. J. Comput. Neurosci. 4, 349-369.

27. Haag J. and Borst A. (1998) Active membrane properties and signal encoding in graded potential neurons. J. Neurosci. 18, 7972-7986.

28. Durr V., Kurtz R., and Egelhaaf M. (2001) Two classes of visual motion sensitive interneurons differ in direction and velocity dependency of in vivo calcium dynamics. J. Neurobiol. 46, 289-300.

29. Gauck V., Egelhaaf M., and Borst A. (1997) Synapse distribution on VCH, an inhibitory, motion-sensitive interneuron in the fly visual system. J. Comp. Neurol. 381, 489-499.

30. van Hateren J. H. (1997) Processing of natural time series of intensities by the visual system of the blowfly. Vision Res. 37, 3407-3416.

31. Weckstrom M. and Laughlin S. B. (1995) Visual ecology and voltage-gated ion channels in insect photoreceptors. Trends Neurosci. 18, 17-21.

32. Laughlin S. B. and Weckstrom M. (1993) Fast and slow photoreceptors-a comparative study of the functional diversity of coding and conductance in the Diptera. J. Comp. Physiol. [A] 172, 593-609. 
33. Mendenhall B. and Murphey R. K. (1974) The morphology of cricket giant interneurons. $J$. Neurobiol. 5, 565-580.

34. Baba Y., Hirota K., Shimozawa T., and Yamaguchi T. (1995) Differing afferent connections of spiking and nonspiking wind-sensitive local interneurons in the terminal abdominal ganglion of the cricket Gryllus bimaculatus. J. Comp. Physiol. [A] 176, 17-30.

35. Jacobs G. A. and Miller J. P. (1985) Functional properties of individual neuronal branches isolated in situ by laser photoinactivation. Science 228, 344-346.

36. Jacobs G. A., Miller J. P., and Murphey R. K. (1986) Integrative mechanisms controlling directional sensitivity of an identified sensory interneuron. J. Neurosci. 6, 2298-2311.

37. Ogawa H., Baba Y., and Oka K. (1996) Dendritic $\mathrm{Ca} 2+$ response in cercal sensory interneurons of the cricket Gryllus bimaculatus. Neurosci. Lett. 219, 21-24.

38. Ogawa H., Baba Y., and Oka K. (1999) Dendritic $\mathrm{Ca} 2+$ transient increase evoked by wind stimulus in the cricket giant interneuron. $\mathrm{Neu}$ rosci. Lett. 275, 61-64.

39. Ogawa H., Baba Y., and Oka K. (2000) Spikedependent calcium influx in dendrites of the cricket giant interneuron. J. Neurobiol. 44, 45-56.

40. Ogawa H., Baba Y., and Oka K. (2001) Dendritic calcium accumulation regulates wind sensitivity via short-term depression at cercal sensory-to-giant interneuron synapses in the cricket. J. Neurobiol. 46, 301-313.

41. Howard J., Blakeslee B., and Laughlin S. B. (1987) The intracellular pupil mechanism and photoreceptor signal: noise ratios in the fly Lucilia cuprina. Proc. R. Soc. Lond B Biol. Sci. 231, 415-435.

42. Laughlin S. B., Howard J., and Blakeslee B. (1987) Synaptic limitations to contrast coding in the retina of the blowfly Calliphora. Proc. R. Soc. Lond B Biol. Sci. 231, 437-467.

43. Laughlin S. B. (1981) A simple coding procedure enhances a neuron's information capacity. Z. Naturforsch. 36c, 910-912.

44. French A. S., Korenberg M. J., Jarvilehto M., Kouvalainen E., Juusola M., and Weckstrom M. (1993) The dynamic nonlinear behavior of fly photoreceptors evoked by a wide range of light intensities. Biophys. J. 65, 832-839.

45. Juusola M. and Weckstrom M. (1993) Bandpass filtering by voltage-dependent mem- brane in an insect photoreceptor. Neurosci. Lett. 154, 84-88.

46. Juusola M., Kouvalainen E., Jarvilehto M., and Weckstrom M. (1994) Contrast gain, signal-tonoise ratio, and linearity in light-adapted blowfly photoreceptors. J. Gen. Physiol. 104, 593-621.

47. Juusola M. and Hardie R. C. (2001) Light adaptation in Drosophila photoreceptors: I. Response dynamics and signaling efficiency at 25 degrees C. J. Gen. Physiol. 117, 3-25.

48. Juusola M. and Hardie R. C. (2001) Light adaptation in Drosophila photoreceptors: II. Rising temperature increases the bandwidth of reliable signaling. J. Gen. Physiol. 117, 27-42.

49. Weckstrom M., Hardie R. C., and Laughlin S. B. (1991) Voltage-activated potassium channels in blowfly photoreceptors and their role in light adaptation. J. Physiol. 440, 635-657.

50. Sobel E. C. and Tank D. W. (1994) In vivo Ca2+ dynamics in a cricket auditory neuron: an example of chemical computation. Science 263, 823-825.

51. Maddess T. and Laughlin S. B. (1985) Adaptation of the motion-sensitive neuron $\mathrm{H} 1$ is generated locally and governed by contrast frequency. Proc. R. Soc. Lond B Biol. Sci. 228, 251-275.

52. Jian S. and Horridge G. A. (1991) The H1 neuron measures change in velocity irrespective of contrast frequency, mean velocity or velocity modulation frequency. Philos. Trans. R. Soc. Lond B Biol. Sci. 331, 205-211.

53. Fairhall A. L., Lewen G. D., Bialek W., and de Ruyter van Steveninck R. R. (2001) Efficiency and ambiguity in an adaptive neural code. Nature 412, 787-792.

54. Harris R. A., O'Carroll D. C., and Laughlin S. B. (2000) Contrast gain reduction in fly motion adaptation. Neuron 28, 595-606.

55. Kurtz R., Durr V., and Egelhaaf M. (2000) Dendritic calcium accumulation associated with direction-selective adaptation in visual motion-sensitive neurons in vivo. J. Neurophysiol. 84, 1914-1923.

56. Harris R. A., O'Carroll D. C., and Laughlin S. B. (1999) Adaptation and the temporal delay filter of fly motion detectors. Vision Res. 39, 2603-2613.

57. Clifford C. W. and Langley K. (1996) A model of temporal adaptation in fly motion vision. Vision Res. 36, 2595-2608.

58. de Ruyter van Steveninck R. R., Zaagman W. H., and Mastebroeck H.A.K. (1986) Adapta- 
tion of transient responses of a movement-sensitive neuron in the visual system of the blowfly Calliophora erythrocephala. Biol. Cybern. 54, 223-236.

59. Durr V. and Egelhaaf M. (1999) In vivo calcium accumulation in presynaptic and postsynaptic dendrites of visual interneurons. $J$. Neurophysiol. 82, 3327-3338.

60. Borst A. and Egelhaaf M. (1992) In vivo imaging of calcium accumulation in fly interneurons as elicited by visual motion stimulation. Proc. Natl. Acad. Sci. USA 89, 4139-4143.

61. Brenner N., Bialek W., and de Ruyter van Steveninck R. R. (2000) Adaptive rescaling maximizes information transmission. Neuron 26, 695-702.

62. Fortune E. S. and Rose G. J. (2000) Short-term synaptic plasticity contributes to the temporal filtering of electrosensory information. J. Neurosci. 20, 7122-7130.

63. Fortune E. S. and Rose G. J. (2001) Short-term synaptic plasticity as a temporal filter. Trends Neurosci. 24, 381-385.

64. Juusola M., Uusitalo R. O., and Weckstrom M. (1995) Transfer of graded potentials at the photoreceptor-interneuron synapse. J. Gen. Physiol. 105, 117-148.

65. Juusola M., French A. S., Uusitalo R. O., and Weckstrom M. (1996) Information processing by graded-potential transmission through tonically active synapses. Trends Neurosci. 19, 292-297.

66. von Gersdorff H. (2001) Synaptic ribbons: versatile signal transducers. Neuron 29, 7-10.

67. Morgans C. W. (2000) Neurotransmitter release at ribbon synapses in the retina. Immunol. Cell Biol. 78, 442-446.

68. Attwell D., Borges S., Wu S. M., and Wilson M. (1987) Signal clipping by the rod output synapse. Nature 328, 522-524.

69. Kurtz R., Warzecha A. K., and Egelhaaf M. (2001) Transfer of visual motion information via graded synapses operates linearly in the natural activity range. J. Neurosci. 21, 6957-6966.

70. Hengstenberg R. (1982) Common visual response properties of giant vertical cells in the lobula plate of the blowfly Calliphora. J. Comp. Physiol. [A] 149, 179-193.

71. Hengstenberg R., Hausen K., and Hengstenberg B. (1982) The number and structure of giant vertical cells (VS) in the lobula plate of the blowfly Calliphora erythrocephala. J. Comp. Physiol. [A] 149, 163-177.
72. Krapp H. G., Hengstenberg B., and Hengstenberg R. (1998) Dendritic structure and receptive-field organization of optic flow processing interneurons in the fly. J. Neurophysiol. 79, 1902-1917.

73. Warzecha A. K., Kurtz R., and Egelhaaf M. (2002) Synaptic transfer of dynamical motion information between identified neurons in the visual system of the blowfly, submitted.

74. Warzecha A. K., Kretzberg J., and Egelhaaf M. (1998) Temporal precision of the encoding of motion information by visual interneurons. Curr. Biol. 8, 359-368.

75. Hausen K. and Egelhaaf M. (1989) Neural mechanisms of visual course control in insects, in Facets of Vision (Stavenga D. G. and Hardie R. C., eds.), Springer, Berlin, Heidelberg, pp. 391-424.

76. Augustine G. J. (2001) How does calcium trigger neurotransmitter release? Curr. Opin. Neurobiol. 11, 320-326.

77. Bollmann J. H., Sakmann B., and Borst J. G. (2000) Calcium sensitivity of glutamate release in a calyx-type terminal. Science 289, 953-957.

78. Heidelberger R., Heinemann C., Neher E., and Matthews G. (1994) Calcium dependence of the rate of exocytosis in a synaptic terminal. Nature 371, 513-515.

79. Qian J. and Noebels J. L. (2001) Presynaptic $\mathrm{Ca} 2+$ channels and neurotransmitter release at the terminal of a mouse cortical neuron. J. Neurosci. 21, 3721-3728.

80. Schneggenburger R. and Neher E. (2000) Intracellular calcium dependence of transmitter release rates at a fast central synapse. Nature 406, 889-893.

81. Smith S. J., Augustine G. J., and Charlton M. P. (1985) Transmission at voltage-clamped giant synapse of the squid: evidence for cooperativity of presynaptic calcium action. Proc. Natl. Acad. Sci. USA 82, 622-625.

82. Wu L. G. and Saggau P. (1994) Presynaptic calcium is increased during normal synaptic transmission and paired-pulse facilitation, but not in long-term potentiation in area CA1 of hippocampus. J. Neurosci. 14, 645-654.

83. Ivanov A. I. and Calabrese R. L. (2000) Intracellular $\mathrm{Ca} 2+$ dynamics during spontaneous and evoked activity of leech heart interneurons: low-threshold Ca currents and graded synaptic transmission. J. Neurosci. 20, 4930-4943.

84. Haag J. and Borst A. (2000) Spatial distribution and characteristics of voltage-gated calcium 
signals within visual interneurons. J. Neurophysiol. 83, 1039-1051.

85. Witkovsky P., Schmitz Y., Akopian A., Krizaj D., and Tranchina D. (1997) Gain of rod to horizontal cell synaptic transfer: relation to glutamate release and a dihydropyridinesensitive calcium current. J. Neurosci. 17, 7297-7306.

86. Gleason E., Borges S., and Wilson M. (1994) Control of transmitter release from retinal amacrine cells by $\mathrm{Ca} 2+$ influx and efflux. Neuron 13, 1109-1117.

87. Beutner D., Voets T., Neher E., and Moser T. (2001) Calcium dependence of exocytosis and endocytosis at the cochlear inner hair cell afferent synapse. Neuron 29, 681-690.

88. von Gersdorff H. (2001) Synaptic ribbons: versatile signal transducers. Neuron 29, 7-10.

89. Stewart B. A., Mohtashami M., Trimble W. S., and Boulianne G. L. (2000) SNARE proteins contribute to calcium cooperativity of synaptic transmission. Proc. Natl. Acad. Sci. USA 97, 13,955-13,960.

90. Brandstatter J. H., Wassle H., Betz H., and Morgans C. W. (1996) The plasma membrane protein SNAP-25, but not syntaxin, is present at photoreceptor and bipolar cell synapses in the rat retina. Eur. J. Neurosci. 8, 823-828.

91. Morgans C. W., Gaughwin P., and Maleszka R. (2001) Expression of the alpha1F calcium channel subunit by photoreceptors in the rat retina. Mol. Vis. 7, 202-209.

92. Passaglia C., Dodge F., Herzog E., Jackson S., and Barlow R. (1997) Deciphering a neural code for vision. Proc. Natl. Acad. Sci. USA 94, $12,649-12,654$.

93. Ilg U. J. and Thier P. (1996) Inability of rhesus monkey area V1 to discriminate between selfinduced and externally induced retinal image slip. Eur. J. Neurosci. 8, 1156-1166.

94. Kern R., Lutterklas M., and Egelhaaf M. (2000) Neuronal representation of optic flow experienced by unilaterally blinded flies on their mean walking trajectories. J. Comp. Physiol. [A] 186, 467-479.

95. Kern R., Lutterklas M., Petereit C., Lindemann J. P., and Egelhaaf M. (2001) Neuronal processing of behaviourally generated optic flow: experiments and model simulations. Network: Comput. Neural Syst. 12, 351-369.

96. Kern R., Petereit C., and Egelhaaf M. (2001) Neural processing of naturalistic optic flow. J. Neurosci. 21, RC139.
97. Kimmerle B. and Egelhaaf M. (2000) Detection of object motion by a fly neuron during simulated flight. J. Comp. Physiol. [A] 186, 21-31.

98. Hateren J. H. and Schilstra C. (1999) Blowfly flight and optic flow. II. Head movements during flight. J. Exp. Biol. 202 (Pt 11), 1491-1500.

99. Schilstra C. and van Hateren J. H. (1998) Using miniature sensor coils for simultaneous measurement of orientation and position of small, fast-moving animals. J. Neurosci. Methods 83, 125-131.

100. Schilstra C. and van Hateren J. H. (1998) Stabilizing gaze in flying blowflies. Nature 395, 654.

101. Schilstra C. and Hateren J. H. (1999) Blowfly flight and optic flow. I. Thorax kinematics and flight dynamics. J. Exp. Biol. 202 (Pt 11), 1481-1490.

102. Kern R., Lindemann J. P., Meyer P., Witte U., and Egelhaaf M. (2001) A new stimulus device for panoramic and highspeed presentation of behaviourally generated optic flow. 6th international congress of neuroethology (abstract), 65.

103. O'Keefe J. (1976) Place units in the hippocampus of the freely moving rat. Exp. Neurol. 51, 78-109.

104. Helmchen F., Fee M. S., Tank D. W., and Denk W. (2001) A miniature head-mounted twophoton microscope. high-resolution brain imaging in freely moving animals. Neuron 31, 903-912.

105. Egelhaaf M. (1985) On the neuronal basis of figure-ground discrimination by relative motion in the visual system of the fly: II. Figure-detection cells, a new class of visual interneurons. Biol. Cybern. 52, 195-209.

106. Hausen K. (1982) Motion sensitive interneurons in the optomotor system of the fly: II. The horizontal cells: receptive field organization and response characteristics. Biol. Cybern. 46, 67-79.

107. Hausen K. (1982) Motion sensitive interneurons in the optomotor system of the fly: I. The horizontal cells: structure and signals. Biol. Cybern. 45, 143-156.

108. Kimmerle B. and Egelhaaf M. (2000) Performance of fly visual interneurons during object fixation. J. Neurosci. 20, 6256-6266.

109. Krapp H. G. and Hengstenberg R. (1996) Estimation of self-motion by optic flow processing in single visual interneurons. Nature 384, 463-466. 